\title{
Re-evaluation of the endometriosis fertility index for predicting spontaneous pregnancy after surgery: a retrospective study
}

\author{
鳥取大学医学部 産科婦人科学分野
}

東 幸弘、谷口文紀、宮本圭輔、中曽崇也、池淵＼cjkstart愛、 森山真亜子、山根恵美子、佐藤絵理、原田 省

\section{Re-evaluation of the endometriosis fertility index for predicting spontaneous pregnancy after surgery: a retrospective study}

\author{
Yukihiro Azuma, Fuminori Taniguchi, Keisuke Miyamoto, Takaya Nakaso, Ai Ikebuchi, \\ Maako Moriyama, Emiko Yamane, Eri Sato and Tasuku Harada \\ Department of Obstetrics and Gynecology, Tottori University Faculty of Medicine, Yonago 683-8504, Japan
}

\begin{abstract}
Purpose: To verify the efficacy of the endometriosis fertility index (EFI) for predicting spontaneous pregnancy after surgery for endometriosis.

Methods: We retrospectively evaluated the predictive value of postoperative EFI for spontaneous pregnancy in 26 patients who underwent surgery for ovarian endometrioma in our institution between 2010 and 2016.

Results: The median EFI score in all patients was 7, and the rate of spontaneous pregnancy after surgery was $35 \%$. The cut-off EFI score obtained from the receiver operating characteristic (ROC) curve was 8 (AUC, 0.79; sensitivity, 0.67; specificity, 0.82). Rate of spontaneous pregnancy with EFI scores $\leq 7$ was significantly lower than that with EFI scores $\geq$ 8 (18\% vs. $67 \%, P=0.028)$. Infertility treatment with assisted reproductive technology (ART) increased pregnancy rates in the lower EFI score group.

Conclusion: We confirmed that the EFI was a reliable prediction tool for spontaneous pregnancy in patients with surgically treated endometriosis. In infertile patients with EFI scores $\leq 7$, ART therapy should be recommended.
\end{abstract}

Key words: endometriosis fertility index, infertility, spontaneous pregnancy

\section{Introduction}

Endometriosis is a benign estrogen-dependent disorder that occurs in approximately $10 \%$ of reproductive-age women. ${ }^{1)}$ Chronic inflammatory status and intrapelvic adhesion cause infertility in endometriosis patients. Moreover, intraperitoneal high cytokine environment observed in endometriosis patients may affect sperm and their interaction with the oocyte. ${ }^{2)}$ Thus, endometriosis can negatively affect the quality of life of patients in terms of both social and reproductive aspects.

Hormonal medication and surgical removal of endometriotic lesions are clinically regarded as the main treatments for endometriosis. Although cystectomy for ovarian endometrioma (OE) is considered a more effective intervention for symptom relief, this approach can cause significant damages to the ovarian reserve. ${ }^{3,4)}$ However, 
Esinler et al. reported that diminished ovarian reserves did not translate into impaired pregnancy outcomes of controlled ovarian hyperstimulation and intracytoplasmic sperm injection (ICSI). ${ }^{5}$ ) The American Society of Reproductive Medicine (ASRM) commented that there is insufficient evidence indicating that resection of endometriotic lesions prior to in vitro fertilization (IVF) improved infertility treatment outcomes. ${ }^{6}$ ) The European Society for Human Reproduction and Embryology (ESHRE) recommended that surgery should be recommended for infertile women with all stages of endometriosis to ameliorate the rate of spontaneous pregnancy, ${ }^{7.8)}$ and that surgery for endometriosis should be carefully performed in cases of recurrent disease. ${ }^{9)}$ Indeed, the clinical indication and approach of surgical excision and infertility treatment are still controversial.

The revised ASRM ( $r$-ASRM) classification has been employed to predict the recurrence potential of endometriosis based on surgical findings, however, it has limited predictive ability for conception. ${ }^{10)}$ Vercellini et al. observed no association between the r-ASRM staging of endometriosis and the cumulative probability of pregnancy. ${ }^{8)}$ Nevertheless, this staging system has been widely applied for decades.

In 2010, Adamson et al. presented a novel classification system, the endometriosis fertility index (EFI), to predict the natural conception of infertile patients with surgically treated endometriosis, which could avoid wasted time and unnecessary treatment in those with poor pregnancy prognosis. ${ }^{11)}$ This index includes both historical (age at surgery, duration of infertility, prior pregnancy history) and surgical factors (the least function score, r-ASRM endometriosis score, and r-ASRM total score) (Figure 1A). The least function score is calculated at the end of the surgery, based on the bilateral morphology of the adnexa, which includes the fallopian tube, fimbria, and ovary (Figure 1B). Morphology of the adnexa, as emphasized in the EFI, is crucial for the prediction of spontaneous pregnancy, since the fallopian tubes play an important role in catching ovulated eggs, and as the site of fertilization.
Japan involves the highest use of assisted reproductive technology (ART) for infertility treatment worldwide. ${ }^{12)}$ Such treatment has been indicated for infertile, surgically-treated endometriosis patients with sufficient potential to conceive naturally. The purpose of our study was to re-evaluate the utility of EFI in Japanese infertile patients with endometriosis.

\section{Materials and Methods}

Study design

We performed laparoscopic ovarian cystectomy for $\mathrm{OE}$ in 43 patients who desired pregnancy after surgery between October 2010 and December 2016. Patients who underwent ART before the surgery and who were not actively trying to conceive were excluded. None of the patients underwent oophorectomy or salpingectomy. All patients were pathologically diagnosed with endometriosis. No cases were administered perior postoperative hormonal medication. Patients with uterine abnormalities, including benign uterine diseases such as uterine fibroids and adenomyosis, were excluded. Conditions such as male factor infertility and polycystic ovary syndrome were also excluded. The period of trying to conceive naturally was set at 2 years after surgery and patients treated by ART within this period were excluded. We retrospectively evaluated the EFI score of 26 patients finally (Figure 2). r-ASRM endometriosis score and r-ASRM total score were calculated by lesion size and adhesion area based on the r-ASRM classification system. ${ }^{13)}$ The overall observation period was set at 3 years after the surgery. Positive pregnancy was defined as detection of gestational sac by transvaginal ultrasonography.

\section{Surgical method}

All patients underwent laparoscopic cystectomy for OE under general anesthesia using a four-port approach after establishment of the pneumoperitoneum via a Veress needle. After visual inspection of the abdomen and pelvis, adhesiolysis was performed to restore normal anatomical position. Stripping of the endometrioma wall from normal ovarian parenchyma was 
(A)

\begin{tabular}{|c|c|c|}
\hline \multicolumn{3}{|c|}{ Historical Factors } \\
\hline Factor & & Points \\
\hline \multirow[t]{3}{*}{ Age } & $\leq 35$ years & 2 \\
\hline & $36-39$ years & 1 \\
\hline & $\geq 40$ years & 0 \\
\hline \multicolumn{3}{|c|}{ Years infertile } \\
\hline & $\leq 3$ & 2 \\
\hline & $>3$ & 0 \\
\hline \multicolumn{3}{|c|}{ Prior Pregnancy } \\
\hline & Yes & 1 \\
\hline & No & 0 \\
\hline \multicolumn{3}{|c|}{ Surgical Factors } \\
\hline Factor & & Points \\
\hline \multirow[t]{3}{*}{ LF score } & $7-8$ & 3 \\
\hline & $4-6$ & 2 \\
\hline & $0-3$ & 0 \\
\hline \multicolumn{3}{|c|}{ r-ASRM Endometrial Score } \\
\hline & $<16$ & 2 \\
\hline & $\geq 16$ & 0 \\
\hline \multicolumn{3}{|c|}{ r-ASRM Total Score } \\
\hline & $<71$ & 1 \\
\hline & $\geq 71$ & 0 \\
\hline & ore is EFI & \\
\hline
\end{tabular}

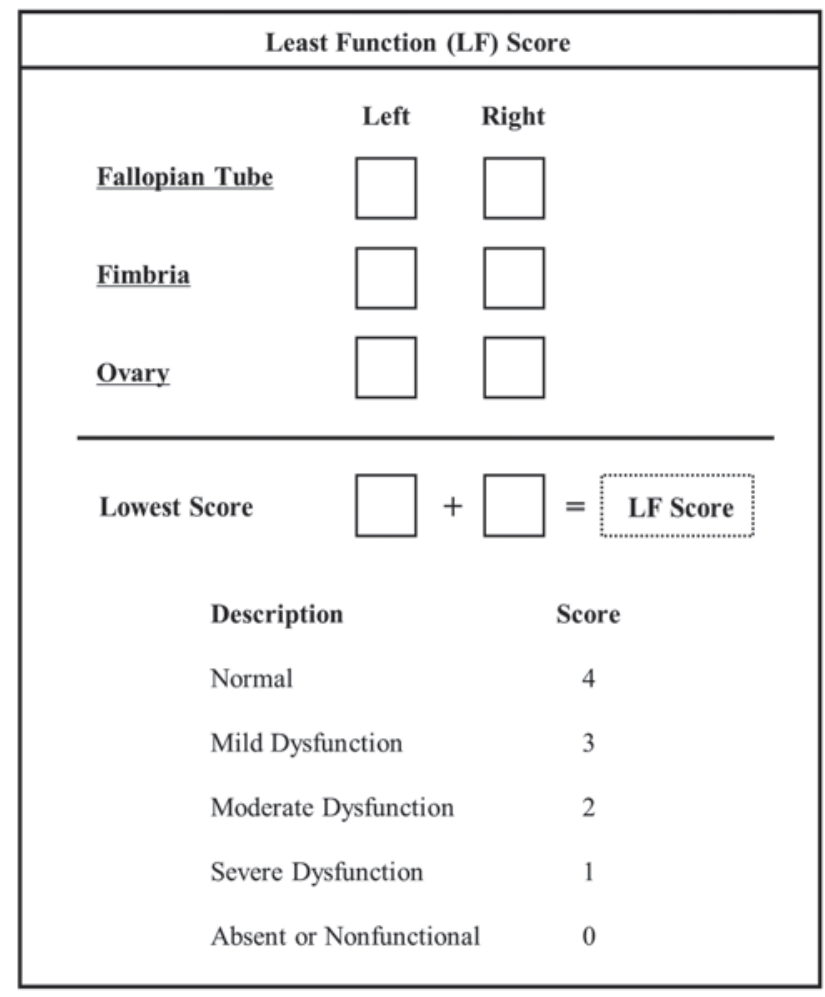

Figure 1. EFI and LF score calculation.

(A) EFI includes both historical factors and surgical factors.

(B) LF score is calculated based on the bilateral morphology of the adnexa.

EFI: endometriosis fertility index, LF score: least function score

performed by gentle countertraction with the use of grasping forceps and scissors. Bleeding was stopped by coagulation with monopolar (ENDOPATH Probe Plus II , Johnson \& Johnson, U.S.A.) or bipolar (ENDOPATH, Johnson \& Johnson, U.S.A.) forceps, which were used as little as possible to avoid heat damage to the normal ovarian tissue. All visible peritoneal endometriotic lesions were excised or coagulated. At the end of surgery, tubal patency was assessed with antidromic injection of indigo carmine. All excised OE cyst walls were histologically diagnosed as endometriosis.

\section{Statistical analysis}

All statistical analyses were performed using the SPSS v25.0 software (IBM, U.S.A.). Nonnormally distributed data are reported as median (minimum-maximum range) and were compared using the Mann-Whitney test. Comparison between the two EFI groups was performed using the Fisher's exact test. Significance was defined as a two-tailed P-value of $\leq 0.05$. The receiver operating characteristic (ROC) curve was used to analyze the predictive values of EFI for natural conception, and accuracy was evaluated in term of sensitivity, specificity, and the Youden's index. The ROC analysis was used to calculate the cut-off point of each index for successful prediction. Kaplan-Meier curves were used to assess the cumulative rates of spontaneous pregnancy at different time points.

\section{Ethical approval, human rights statements, and informed consent}

This retrospective study was conducted in the Department of Obstetrics and Gynecology, Tottori University Hospital, Tottori, Japan, after receiving Institutional Review Board approval 


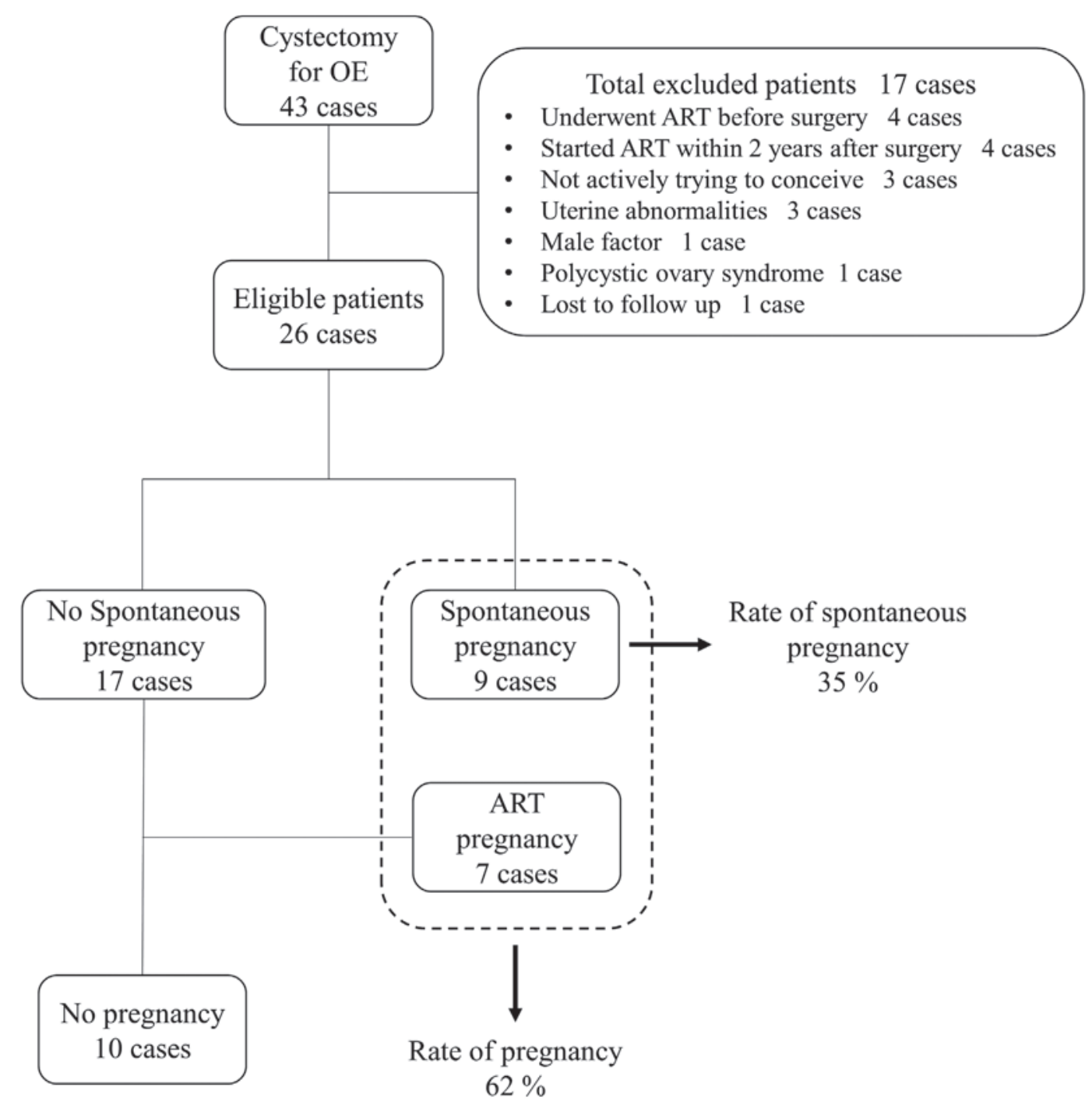

Figure 2. Pregnancy outcomes after surgery for endometriosis.

Sixteen patients achieved pregnancy within 3 years after surgery. Nine of them conceived spontaneously (34.6\%), while the others needed ART treatment.

OE: ovarian endometrioma, ART: assisted reproductive technology.

(approval number: 20A037). All procedures followed were in accordance with the Helsinki Declaration of 1964 and its later amendments. Written informed consent for the retrospective analysis and inclusion of de-identified data was obtained from all patients in the study.

\section{Results}

Characteristics of all 26 patients are summarized in Table 1 . The median age at surgery for endometriosis was $32(25-40)$ years. The duration of infertility before surgery was $\leq 3$ years in most cases (96\%), and over half of the patients were nulliparous. The median least function score was 5 , and 9 patients (35\%) were classified as the low score group. The median r-ASRM endometriosis score and total score were 26 and 35, respectively, and almost all patients were diagnosed with ASRM Stage III / IV endometriosis.

Sixteen patients became pregnant within 3 years after cystectomy for OE (Rate of pregnancy, $62 \%$ ). Nine of them conceived naturally (Rate of spontaneous pregnancy, 35\%), while the others needed ART treatment (Figure 2). No incidence of miscarriage was reported during the study period. Data of the two groups categorized as spontaneous and assisted pregnancy groups are presented in Table 2. As expected, the EFI score of the spontaneous pregnancy group was significantly higher than that of the assisted 
Table.1 Patient characteristics

\begin{tabular}{ll}
\hline Age at surgery (years) & $32(25-40)$ \\
$\leq 35$ & $21(81 \%)$ \\
$36-39$ & $4(15 \%)$ \\
$\geq 40$ & $1(4 \%)$ \\
& \\
Duration of infertility (years) & $1(0-3)$ \\
$\leq 3$ & $25(96 \%)$ \\
$>3$ & $1(4 \%)$ \\
& \\
History of prior pregnancy & \\
$\quad$ & \\
- & $11(42 \%)$ \\
Least function score & $15(58 \%)$ \\
High (7-8) & \\
Moderate $(4-6)$ & $5(1-8)$ \\
Low (1-3) & $6(23 \%)$ \\
& $11(42 \%)$ \\
r-ASRM endometriosis lesion score & $9(35 \%)$ \\
$<16$ & \\
$\geq 16$ & $26(18-50)$ \\
r-ASRM total score & $0(0 \%)$ \\
$<71$ & $26(100 \%)$ \\
$\geq 71$ & \\
\hline
\end{tabular}

Data were shown as the median (min-max) or patient number (percentage).

Table.2 Patients characteristics classified by spontaneous pregnancy after surgery

\begin{tabular}{|c|c|c|c|}
\hline & \multicolumn{2}{|c|}{ Spontaneous pregnancy } & \multirow[b]{2}{*}{ Significance } \\
\hline & $\begin{array}{l}\text { Yes } \\
(n=9)\end{array}$ & $\begin{array}{l}\text { No } \\
(\mathrm{n}=17)\end{array}$ & \\
\hline EFI & $8(5-9)$ & $6(4-9)$ & $P=0.015$ \\
\hline Age at surgery (years) & $31(27-34)$ & $34(25-40)$ & $P=0.14$ \\
\hline \multicolumn{4}{|l|}{ Duration of infertility (years) } \\
\hline$\leq 3$ & $9(100 \%)$ & $16(94 \%)$ & $P=1.000$ \\
\hline$>3$ & $0(0 \%)$ & $1(6 \%)$ & \\
\hline \multicolumn{4}{|l|}{ History of prior pregnancy } \\
\hline+ & $4(44 \%)$ & $7(41 \%)$ & $P=1.000$ \\
\hline- & $5(56 \%)$ & $10(59 \%)$ & \\
\hline Least function score & $6(3-7)$ & $4(1-7)$ & $P=0.034$ \\
\hline r-ASRM endometriosis lesion score & $26(18-35)$ & $36(18-50)$ & $P=0.081$ \\
\hline r-ASRM total score & $37(19-48)$ & $53(22-130)$ & $P=0.067$ \\
\hline
\end{tabular}

Data were shown as the median (min-max) or patient number (percentage). 
Table.3 Comparison of pregnancy outcome by EFI score

\begin{tabular}{llll}
\hline & $\begin{array}{l}\text { EFI: 0-7 } \\
(\mathrm{n}=17)\end{array}$ & $\begin{array}{l}\text { EFI: 8-10 } \\
(\mathrm{n}=9)\end{array}$ & Significance \\
\hline Median age (years) & $33(25-40)$ & $31(27-34)$ & $P=0.24$ \\
Rate of spontaneous pregnancy (\%) & 18 & 67 & $P=0.028$ \\
$\begin{array}{l}\text { Overall pregnancy rate including } \\
\text { ART (\%) }\end{array}$ & 53 & 78 & $P=0.39$ \\
\hline
\end{tabular}

(A)

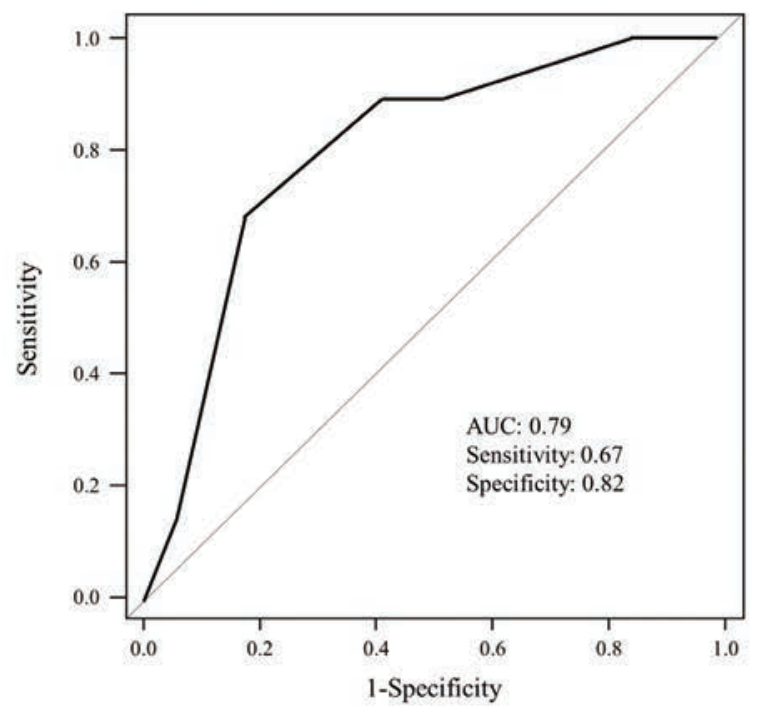

(B)

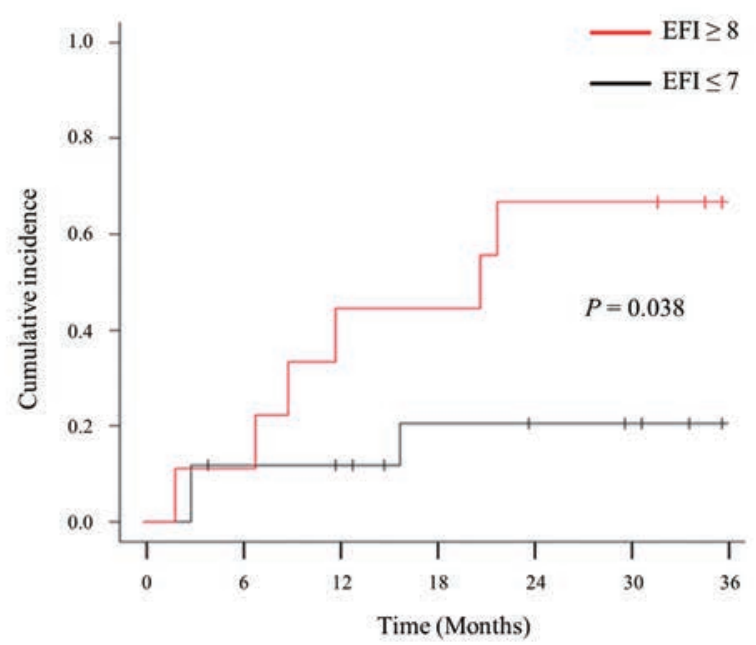

Figure 3. Receiver operating characteristic $(\mathrm{ROC})$ curve and cumulative rate of spontaneous pregnancy after surgery for endometriosis.

(A) The optimal cut-off EFI score derived from the ROC curve was 8 (sensitivity, 0.67; specificity, 0.82). The area under the curve (AUC) for EFI as the predictor of pregnancy was $0.79(95 \% \mathrm{Cl}, 0.616-0.971)$.

(B) Patients with EFI scores $\geq 8$ had significantly higher cumulative rates of spontaneous pregnancy compared to those with EFI scores $\leq 7$.

pregnancy group (median, 8 vs. $6 ; P=0.015$ ). Among the constituent variables of EFI, only the least function score was significantly different between the two groups ( 6 vs. $4 ; P=0.034$ ). The median EFI score was 7 (4-9) in all 26 patients.

As shown in Figure 3A, the area under the ROC curve (AUC) for the prediction of natural conception was 0.79 (95\% CI, 0.616-0.971). The optimal cut-off EFI score was 8 (sensitivity, 0.67; specificity, 0.82 ). Patients were classified into two groups, the high $\mathrm{EFI}(\geq 8)$ and low $\mathrm{EFI}(\leq 7)$ group, and the cumulative rates of spontaneous pregnancy in each group are shown in Figure 3B. Patients with high EFI scores presented significantly higher cumulative rates of spontaneous pregnancy compared with those in the low EFI group $(P=$
0.038).

The median age, as well as the rate of spontaneous and overall pregnancy of each EFI group are exhibited in Table 3. Inferior pregnancy rates of the low EFI group was shown to be rescued by ART therapy to an equivalent level with the high EFI group. Natural conception was not reported in any of the patients aged over 35 years during the observation period.

\section{Discussion}

Our data demonstrated an association between EFI and spontaneous pregnancy in relatively young patients with advanced ASRM stages of endometriosis. We thereby confirmed that the EFI is an effective prediction tool for spontaneous 
pregnancy after endometriosis surgery.

Endometriosis negatively impacts fertility through several ways, including decreased ovarian function, fallopian tube damage secondary to intraperitoneal adhesions, and the chronic inflammatory environment in the pelvis. ${ }^{14)}$ The least function score is considered more important than other elements of the EFI, since morphology of the adnexa plays a crucial role in achieving spontaneous pregnancy. ${ }^{15)}$ While surgery for endometriosis primarily aims to remove endometriotic lesions, it is also important to assess the adnexa and restore normal pelvic anatomy. Few reports of high-quality studies currently exist regarding the issue of whether surgery improves fertility. Demirol et al. reported that cystectomy for OE before ICSI resulted in longer ovarian stimulation with gonadotrophins, higher follicular stimulating hormone requirement, and lower oocyte number, but fertilization, pregnancy, and implantation rates did not differ from those of the patients who underwent ICSI directly. ${ }^{16)}$ Tsoumpou et al. also reported in their systematic review that cystectomy for OE prior to IVF did not improve clinical pregnancy rate or ovarian response to stimulation with gonadotrophins compared with no treatment. ${ }^{17)}$ In the metaanalysis by Barnhart et al., the chance of achieving pregnancy was significantly lower for endometriosis patients compared with tubal factor controls, while pregnancy rates in patients with severe endometriosis were significantly lower than the rates in those with mild disease. ${ }^{18)}$ In contrast to these results, Jacobson et al. demonstrated that laparoscopic surgery for the treatment of subfertility related to minimal and mild endometriosis could improve future fertility. ${ }^{19)}$ Hart et al. suggested that excisional surgery for OE provided more favorable spontaneous pregnancy outcomes than drainage and ablation in previously subfertile women. ${ }^{7}$ Since several reports have shown the favorable cumulative rates of spontaneous pregnancy after cystectomy for $\mathrm{OE}^{7,20)}$ surgical therapy could be considered for the young patients whose ovarian function is sufficient. However, owing to the fear of decline in ovarian function, cystectomy for $\mathrm{OE}$ tends to be avoided. ${ }^{3,4)}$ When evaluating the effects of surgery, it is therefore important to thoroughly evaluate the benefits and disadvantages to the patients.

Several studies have shown the role of EFI in the selection of treatment approaches for infertile patients. Tomassetti et al. reported presented a significant relationship between EFI and the time to achieving pregnancy without ART, with Kaplan-Meier estimates of cumulative overall pregnancy rate being $46 \%$ at 12 months after surgery, ranging from $17 \%$ for EFI scores $0-3$, to $63 \%$ for EFI scores $9-10 .{ }^{21)}$ Likewise, they indicated the least function score as the most important contributor to the EFI score. Boujenah et al. also reported that the least function score significantly associated with spontaneous pregnancy. ${ }^{22)}$ Similarly, in our analysis, only the median least function score of the spontaneous conception group was significantly higher than that of the assisted pregnancy group. No differences in other elements including the r-ASRM scores were observed between the two groups. Therefore, we also considered the least function score as the most important of the EFI.

Determining the cut-off EFI score for predicting spontaneous pregnancy is crucial to provide beneficial advice for patients. An EFI cut-off score of 7 or 8 has been reported by several studies. ${ }^{23,24)}$ Our study corroborated with this, and determined an effective cut-off EFI score of 8 . The AUC in the present study was comparable to the pooled AUC reported in the systematic review by Vesali et al. $(79 \%$ vs. $71 \%)$, ${ }^{25)}$ indicating that EFI provides a greater predictive value for spontaneous pregnancy after surgery. Considering the small cohort size included in our study, larger samples are warranted to determine better cut-off EFI scores for pregnancy prediction in surgicallytreated endometriosis patients.

Boujenah et al. reported that the pregnancy rate without $\mathrm{ART}$ in patients with EFI scores $\geq$ 7 was $48 \%$. In a systematic review by Vesali et al., cumulative non-ART pregnancy rates of 55\% and $69 \%$ were reported in patients with EFI scores 7-8 and 9-10, respectively. ${ }^{25)}$ In line with previous studies, we demonstrated that the 
cumulative rate of spontaneous pregnancy in patients with EFI scores $\geq 8$ was $67 \%$. Zhang et al. suggested that ART treatment should be recommended to patients with failed natural conception two years after surgery, including those with high EFI scores, since most pregnancies were achieved within two years of surgery in their study. ${ }^{23)}$ We noted that the rate of spontaneous pregnancy did not increase markedly one year after surgery. Regardless of the EFI score, if the patient failed to conceive spontaneously within one year after surgery, ART should be strongly recommended. Thus, we recommend the immediate initiation of ART therapy in all patients over 35 years of age after surgery regardless of the EFI score.

The present study had several strengths. The first was the exclusion of influence from uterine morphological abnormalities such as uterine myoma or adenomyoma, which can cause infertility, even though the EFI does not account for uterine morphology. Second, all cases underwent cystectomy for $\mathrm{OE}$ and were histologically diagnosed with endometriosis. This report did not include patients with early stage endometriosis, such as those with superficial lesions or adhesions only; thus, the utility of EFI in patients suffering from infertility due to severe endometriosis could be evaluated.

Several limitations in our study should be acknowledged. First, the EFI staging system does not account for ovarian reserve status and does not reflect surgical skills and techniques. Ovarian cystectomy for OE inevitably induces the reduction of ovarian reserve due to partial shaving of the normal ovarian parenchyma. ${ }^{26,27)}$ Anti-Müllerian hormone (AMH) produced by the granulosa cells of primary to small antral follicles is widely used to measure the ovarian reserve of infertile patients. We previously reported that serum AMH levels after cystectomy for OE decreased markedly. ${ }^{4}$ If we had effectively combined EFI with the evaluation of pre- and postoperative AMH levels, the accuracy of our evaluations could have been improved. Second, due to the recent avoidance of surgery for OE, the number of recruited patients was insufficient to analyze EFI. Moreover, we were unable to examine the utility of the EFI score in ASRM Stage I/II since majority of our patients belonged to ASRM Stage III/IV. Prospective validation is hence needed in the future.

Despite these limitations, the EFI scoring system is potentially beneficial for the prediction of spontaneous pregnancy in infertile women with surgically treated endometriosis.

\section{Acknowledgements}

We would like to thank Editage (www. editage.com) for English language editing.

\section{Conflict of interest}

The authors declare no conflicts of interest.

本論文の要旨は第59回日本産科婦人科内視鏡学 会において発表した。

\section{References}

1) Vercellini P, et al.: Endometriosis: pathogenesis and treatment. Nat Rev Endocrinol. 2014; 10(5): 261-75.

2 ) Harada T, Iwabe T, Terakawa N.: Role of cytokines in endometriosis. Fertil Steril. 2001; 76(1): 1-10.

3 ) Raffi F, Metwally M, Amer S.: The impact of excision of ovarian endometrioma on ovarian reserve: a systematic review and meta-analysis. J Clin Endocrinol Metab. 2012; 97(9): 3146-54.

4 ) Taniguchi F, et al.: Analysis of pregnancy outcome and decline of anti-Müllerian hormone after laparoscopic cystectomy for ovarian endometriomas. J Obstet Gynaecol Res. 2016; 42(11): 1534-40.

5 ) Esinler I, et al.: Outcome of in vitro fertilization/ intracytoplasmic sperm injection after laparoscopic cystectomy for endometriomas. Fertil Steril. 2006; 85(6): $1730-5$

6 ) Practice Committee of the American Society for Reproductive Medicine. Endometriosis and infertility: a committee opinion. Fertil Steril. 2012; 98(3): 591-8.

7 ) Hart RJ, et al.: Excisional surgery versus ablative surgery for ovarian endometriomata. Cochrane Database Syst Rev. 2008; (2): CD004992.

8 ) Vercellini P, et al.: Reproductive performance, pain recurrence and disease relapse after conservative surgical treatment for endometriosis: the predictive value of the current classification system. Hum Reprod. 2006; 21(10): 2679-85.

9) Dunselman GA, et al.: ESHRE guideline: 
management of women with endometriosis. Hum Reprod. 2014; 29(3): 400-12.

10) Guzick DS, et al.: Prediction of pregnancy in infertile women based on the American Society for Reproductive Medicine's revised classification of endometriosis. Fertil Steril. 1997; 67(5): 822-9.

11) Adamson GD, Pasta DJ.: Endometriosis fertility index: the new, validated endometriosis staging system. Fertil Steril. 2010; 94(5): 1609-15. PMID: 19931076

12) Kushnir VA, et al.: Systematic review of worldwide trends in assisted reproductive technology 20042013. Reprod Biol Endocrinol. 2017; 15(1): 6.

13) Revised American Fertility Society classification of endometriosis: 1985. Fertil Steril. 1985; 43(3): 351-2.

14) de Ziegler D, Borghese B, Chapron C.: Endometriosis and infertility: pathophysiology and management. Lancet. 2010; 376(9742): 730-8

15) Maheux-Lacroix S, et al.: Endometriosis fertility index predicts live births following surgical resection of moderate and severe endometriosis. Hum Reprod. 2017; 32(11): 2243-9

16) Demirol A, et al.: Effect of endometrioma cystectomy on IVF outcome: a prospective randomized study. Reprod Biomed Online. 2006; 12(5): 639-43.

17) Tsoumpou I, et al:: The effect of surgical treatment for endometrioma on in vitro fertilization outcomes: a systematic review and meta-analysis. Fertil Steril. 2009; 92(1): 75-87.

18) Barnhart K, Dunsmoor-Su R, Coutifaris C.: Effect of endometriosis on in vitro fertilization. Fertil Steril. 2002; 77(6): 1148-55.

19) Jacobson $T Z$, et al.: Laparoscopic surgery for subfertility associated with endometriosis. Cochrane Database Syst Rev. 2010; (1): CD001398.

20) Alborzi S, et al:: A prospective, randomized study comparing laparoscopic ovarian cystertomy versus denestration and coaglation in patients with endometriomas. Fertil Steril. 2004; 82(6): 1633-7

21) Tomassetti $C$, et al.: External validation of the endometriosis fertility index (EFI) staging system for predicting non-ART pregnancy after endometriosis surgery. Hum Reprod. 2013; 28(5): $1280-8$.

22) Boujenah J, et al.: External validation of the Endometriosis Fertility Index in a French population. Fertil Steril. 2015; 104(1): 119-23.e1.

23) Zhang $X$, et al.: Prediction of Endometriosis Fertility Index in patients with endometriosis-associated infertility after laparoscopic treatment. Reprod Biomed Online. 2018; 37(1): 53-9.

24) Hobo R, et al: The endometriosis fertility index is useful for predicting the ability to conceive without assisted reproductive technology treatment after laparoscopic surgery, regardless of endometriosis. Gynecol Obstet Invest. 2018; 83(5): 493-8.

25) Vesali S, et al: Endometriosis Fertility Index for predicting non-assisted reproductive technology pregnancy after endometriosis surgery: a systematic review and meta-analysis. BJOG. 2020; 127(7): 800-9.

26) Muzii L, et al.: Laparoscopic stripping of endometriomas: a randomized trial on different surgical techniques. Part II: pathological results. Hum Reprod. 2005; 20(7): 1987-92.

27) Muzii L, et al.: Histologic analysis of specimens from laparoscopic endometrioma excision performed by different surgeons: does the surgeon matter? Fertil Steril. 2011; 95(6): 2116-9. 